\title{
A pilot application of Korotkoff sound delay time in evaluating cardiovascular status
}

\author{
Haiyan Xiang*, Yanyong Liu, Yufei Qin, Zhengtao Cao, Tao Guo and Mengsun Yu \\ Aviation Medicine Engineering Centre, Institute of Aviation Medicine, Beijing, China
}

\begin{abstract}
.
BACKGROUND: Korotkoff sounds have been used to measure systolic and diastolic arterial blood pressures noninvasively for over 100 years. However, most of the research concerning the Korotkoff sound were focused on the origin and frequency component analyzing the Korotkoff sound signal.

OBJECTIVE: To show that the occurrence time of the Korotkoff sounds for each cardiac cycle demonstrates a characteristic value during the cuff deflating process of blood pressure measurement.

METHODS: The Korotkoff sound delay time (KDT) decreases as the cuff pressure P deflates and KDT is a function of arterial transmural pressure. In the present research, an experiment system was established to explore the relationship between the KDT and the cuff pressure in different subjects.

RESULTS: A pilot experiment was conducted to obtain different subjects' KDTs and investigate the relationship between KDT and cuff pressure.

CONCLUSION: The relationship between KDT and invasive blood pressure was also studied and its potential application in detection of cardiovascular status was discussed.
\end{abstract}

Keywords: Cardiovascular, noninvasive measurement, Korotkoff sound delay time, transmural pressure

\section{Introduction}

Korotkoff sounds have been used to measure systolic and diastolic arterial blood pressures noninvasively for over 100 years [1-3]. Nicolai Korotkoff first discovered the sounds in the compressed brachial artery and used them to identify the times when the pressure in the cuff coincided with systolic and diastolic arterial pressures. The method utilizing Korotkoff sounds to determine the systolic and diastolic arterial pressure is known as auscultatory method, which is most frequently used in the clinical medicine since 1905 [13].

In the last years, most of the previous researches about Korotkoff sound were focused on the origin and frequency component analyzing of the Korotkoff sounds signal [45]. Some other researchers utilized the Korotkoff sound to replace the distal pulse in the peripheral artery and the QRS wave in electrocardiogram to obtain the pulse transit time (PTT), which was named QKd interval [6]7]. Although the timing of the Korotkoff sound at different cuff pressure was observed by some researchers, the techniques have been only used to improve the electronical auscultatory blood pressure measurement with microphone by electrocardiogram-gating and oscillometric-pulse-gating in reducing the effects of interference and

\footnotetext{
${ }^{*}$ Corresponding author: Haiyan Xiang, Aviation Medicine Engineering Centre, Institute of Aviation Medicine, 28\#, Fucheng Road, Haidian, Beijing 100142, China. Tel.: +86 010 66927045; Fax: +86 010 68453059; E-mail: xianghaiyen@ gmail.com. 
noise for Korotkoff sounds [8]. Further researches related to the occurrence time of the Korotkoff sound at different cuff pressure were not found. In the present research, an experiment system was established to explore the relationship between the Korotkoff sound delay time (KDT) and the cuff pressure in different subjects. A pilot experiment was conducted to obtain different subjects' KDTs and the relationship between KDT and cuff pressure was investigated. The relationship between KDT and invasive blood pressure was also studied and its potential application in detection of cardiovascular status was discussed.

\section{Materials and methods}

\subsection{Components of the system}

The experimental system is similar to that in [9] It includes four units: i) an analog circuit unit to condition the cuff pressure, oscillometric pulse, Korotkoff sounds and invasive blood pressure (IBP); ii) a microprocessor (C8051F060) to receive the analog signals and send them to the computer; iii) a set of automatic inflating and deflating appliance controlled by the microprocessor to realize the linear deflation of the cuff; iv) a personal computer with self-programmed data acquiring and analyzing software [9].

The air channel of the experiment system includes the cuff bladder, the sphygmomanometer, the pressure transducer (Fujikura FPM-07PG), the inflating pump (SEIKO P05105R) and the deflating valve. They are all connected together. The cuff pressure is sensed by the transducer and the amplified pressure signal is fed to the A/D converter of the microprocessor to digitize the pressure in the cuff. The oscillometric pulses of the cuff, high frequency component of the pressure signal in the cuff, are obtained through a high-pass filter (HPF) with cuff-off frequency of $0.3 \mathrm{~Hz}$. The Korotkoff sound transducer (Shengwangsheng MPA201) and the stethoscope bell (3 M Littman 2144 L) are connected together by a tube. Since the Korotkoff sound's energy concentrates mainly below $400 \mathrm{~Hz}$, the passband of the bandpass filter (BPF) for Korotkoff sound channel is $16 \mathrm{~Hz}-400 \mathrm{~Hz}$ [2]5|10-13] The invasive blood pressure transducer (Yixinda DPT-248) is connected to the cannula to measure the pressure of the brachial artery. To avoid aliasing distortion, the four signals then pass a low-pass filter (LPF) with a cut-off frequency of $500 \mathrm{~Hz}$ before starting A/D converting. The microprocessor converts the analog signal to digital signal (12-bits resolution, sample rate $1 \mathrm{kHz}$ for each channel) and sends the acquired data to the computer.

\subsection{Korotkoff sound delay time}

When the auscultatory method is applied to measure arterial pressure, the cuff must be inflated until the pressure in the cuff exceeds systolic pressure. The brachial artery is compressed by the cuff with high pressure. Hence, the artery is occluded completely and there is no blood flow in the brachial artery. Then the cuff deflates smoothly and the cuff pressure drops at a rate of $2-3 \mathrm{mmHg} / \mathrm{sec}$. When the cuff pressure drops somewhat under the systolic blood pressure, the first Korotkoff sound is heard. As the pressure in the cuff decreases from supra-systolic to sub-diastolic, the Korotkoff sounds demonstrates five phases. The appearance and disappearance of the Korotkoff sounds usually were applied to measure the systolic blood pressure (SBP) and the diastolic blood pressure (DBP) respectively during the cuff deflating process.

The Korotkoff sound delay time is defined as the interval from the proximal point to the onset of the current Korotkoff sound. The reference point can be located proximal to the cuff, such as the ECG R 


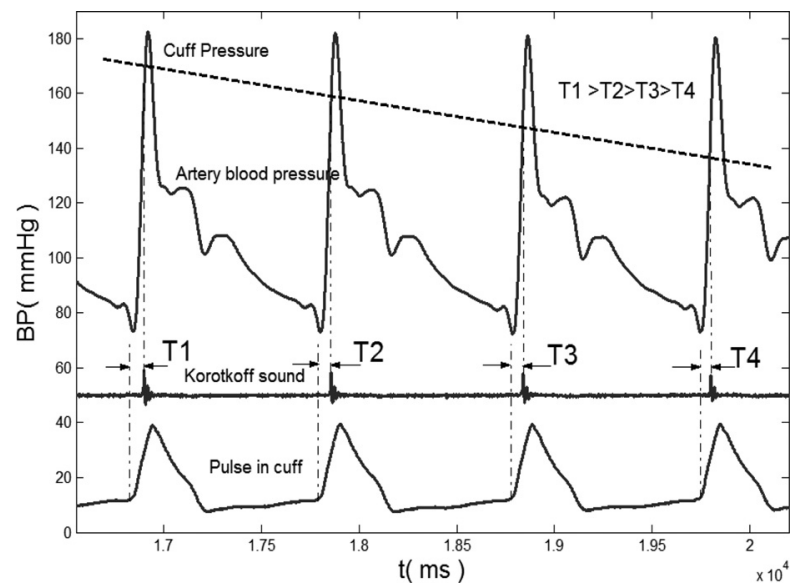

Fig. 1. The Korotkoff sound delay time.

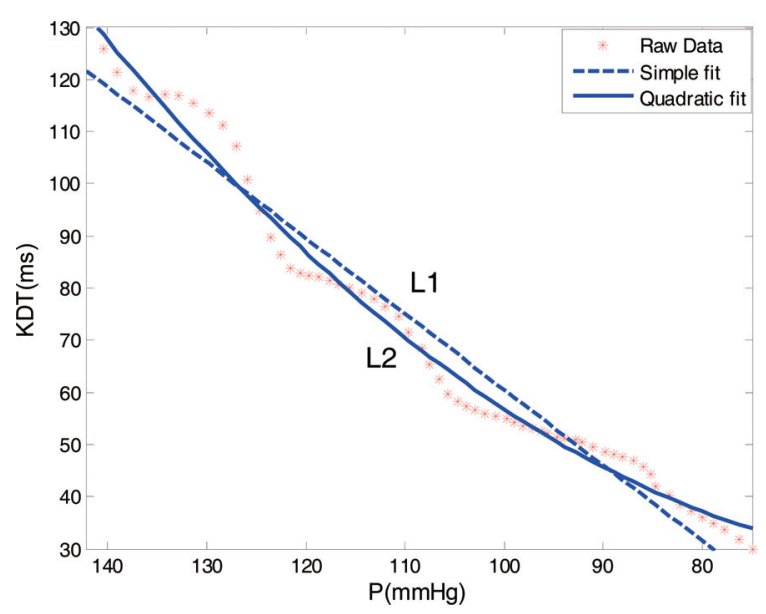

Fig. 2. The KDT and the cuff pressure $P$ in a whole measurement process.

wave, the arterial pulse on the artery between aortic and brachial artery or the ascending edge of pulse wave in the cuff. As shown in Fig. 1. All the time reference point (ECG R wave, arterial pulse and oscillometric pulse in cuff) and the Korotkoff sound are induced by the same systole. In this paper, the ascending edge of the pulse in the cuff is used as the reference point. The Korotkoff sounds delays as cuff pressure increases. As shown in Fig. 2, the Korotkoff sound delay time for the four cardiac cycles at different cuff pressure demonstrate that $\mathrm{T} 1$ at is the longest while T4 is the shortest. In a series of the KDTs of the whole measurement in cuff deflation, the KDT corresponding to the first Korotkoff sound is the longest, while the KDTs corresponding to the subsequent Korotkoff sound become shorter and shorter respectively, and the shortest one appears at the last Korotkoff sound [9].

\subsection{Korotkoff sound delay time for different subjects}

Six subjects' cuff pressure signals and Korotkoff sound signals were acquired simultaneously to explore the characteristics of KDTs for different subjects. The Korotkoff sound delay times of the subjects were computed based on the acquired data in the blood pressure measurement using the above experiment system. The process of the measurement is similar to the standard blood pressure measurement process. The subject is kept quiet for five minutes. The cuff pressure deflates linearly with an approximation ratio of $2 \mathrm{mmHg} / \mathrm{s}$. Based on the linear deflation, the Korotkoff sound delay times were easy to compute and the slow deflation ratio ensure enough cardio cycles in the whole measurement. The Korotkoff sound delay times were plotted against the cuff pressure at each heart and the KDT-P function was obtained. The KDTs for different subjects were calculated. Also the KDTs at different blood pressure status for the same subject were recorded and analyzed. The KDTs for each cardiac cycle are calculated by the oscillometric pulses and the Korotkoff sounds according to the method described in Fig. 1. To obtain the function $f(P)$ of KDT and cuff pressure, the raw KDT data were fitted, as shown in Fig. 2. The "*" represents raw data of KDT. The dashed line represents simple fit and the solid line represents quadratic fit.

\subsection{Korotkoff sound delay time and blood pressure}

The relationship between Korotkoff sound delay time and blood pressure was investigated. The pro- 
cess of the experiment was the same with that in [9]. The cuff pressure, oscillometric pulse and Korotkoff sounds were collected on the subject's right upper arm. The invasive arterial pressure of the left brachial artery was acquired by the IBP transducer and the cannula. The KDTs for each cardiac cycle were calculated by the oscillometric pulses and the Korotkoff sounds. To obtain the obvious relationship between Korotkoff sound delay time and invasive blood pressure, the subject perform deep breathing for approximate $80 \mathrm{~s}$ while the cuff pressure was kept approximate stable near mean arterial pressure. The Korotkoff sound delay times then were calculated and analyzed with the invasive blood pressure recorded simultaneously. The relationship coefficient between KDT and invasive blood pressure was calculated. Moreover, a method to estimate the beat-to-beat mean arterial pressure by Korotkoff sound delay time has been proposed in [9].

\section{Results}

The Korotkoff sound delay times change with the cuff pressure. Figure 2 shows an example of the KDT in the deflation process of the cuff. While the cuff pressure decreases from the supra-systolic pressure to the sub-diastolic pressure, the KDT becomes more and more short. The KDT decreases as the cuff pressure drops. The function relationship which is obtained from a series of KDT and their corresponding cuff pressure $P$ in a whole measurement process can be drawn as a line approximation. The curve L1 is a straight approximation for the KDT. The curve L2 is a quadratic line approximation for a series of KDT. It can be seen that the curve L2 is more approximate to all the KDTs. The function $f(P)$ for $\mathrm{L} 2$ can be described as:

$$
\mathrm{KDT}=f(P)=k_{1} P^{2}+k_{2} P+k_{3}
$$

The "*” represents raw KDT for each heart beat at the corresponding cuff pressure and the solid line is the quadratic fitting curve $\left(\mathrm{KDT}=0.0119 P^{2}-1.1071 P+36.2695\right)$. The raw KDT fluctuates along the fitted curve L2.

Calculate the difference value of the fitted curve L2 obtained in Eq. (1), and then get $g(P)$ :

$$
g(P)=\frac{d \mathrm{KDT}}{d P}=2 k_{1} P+k_{2}
$$

$g(P)$ is the change in KDT for $1 \mathrm{mmHg}$.

The relationship between KDT and cuff pressure demonstrates differently for the six subjects. Figure 3 shows an example of the relationships between KDT and the corresponding cuff pressure for two subjects. The "*" and "+" indicate the raw KDT for each heartbeat of the subjects respectively. The dashed line indicates the function of the lower blood pressure subject, and the solid line indicates the function of the higher blood pressure subject. The left of Fig. 3 indicates the relationship between KDT and the cuff pressure. The right plot of Fig. 3 indicates the relationship between $g(P)$ and the cuff pressure. For the two subjects, the KDT decrease from about $120 \mathrm{~ms}$ at the higher cuff pressure to about $30 \mathrm{~ms}$ at the lower cuff pressure. The $g$ is monotone increasing for both subjects. The maximum $g$ emerge at systolic pressure and the minimum $g$ emerge at diastolic. The $g$ changes from $2.5 \mathrm{~ms} / \mathrm{mmHg}$ to $0.5 \mathrm{~ms} / \mathrm{mmHg}$. The $g$ for the two subjects have different slope. For the subject A, the $g(P)$ is bigger than that of the subject B when the cuff pressure is low. However, when the cuff pressure becomes high, the $g(P)$ of the subject A becomes smaller than that of subject B. To compare the $g(P)$ of different subject, the $g(P)$ at 

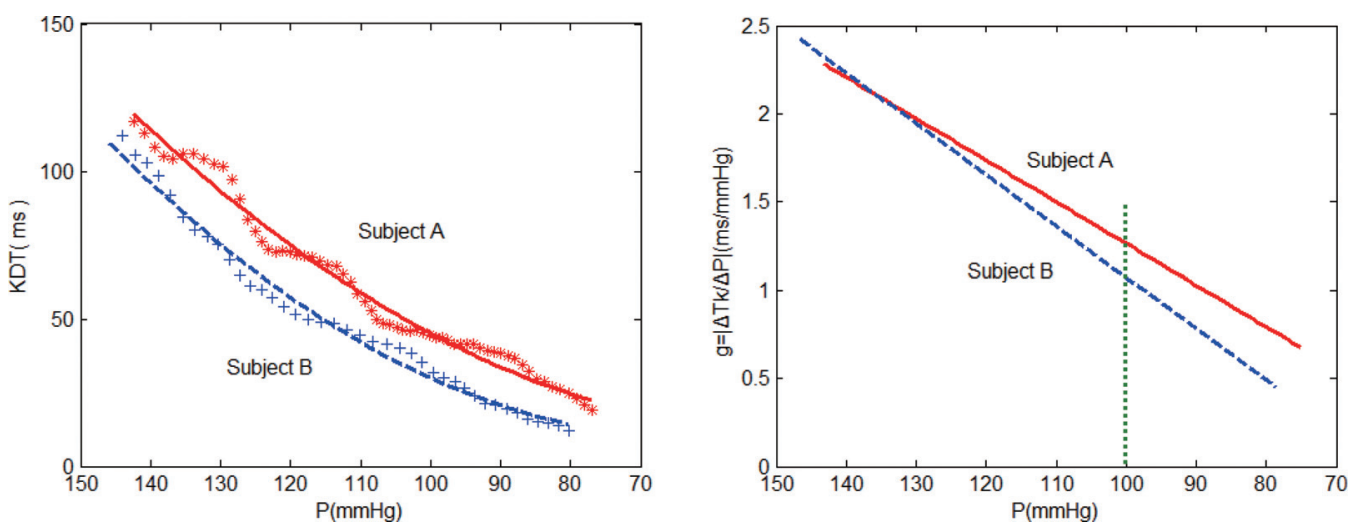

Fig. 3. $\mathrm{KDT}(P)$ and $g(P)$ of different subjects.
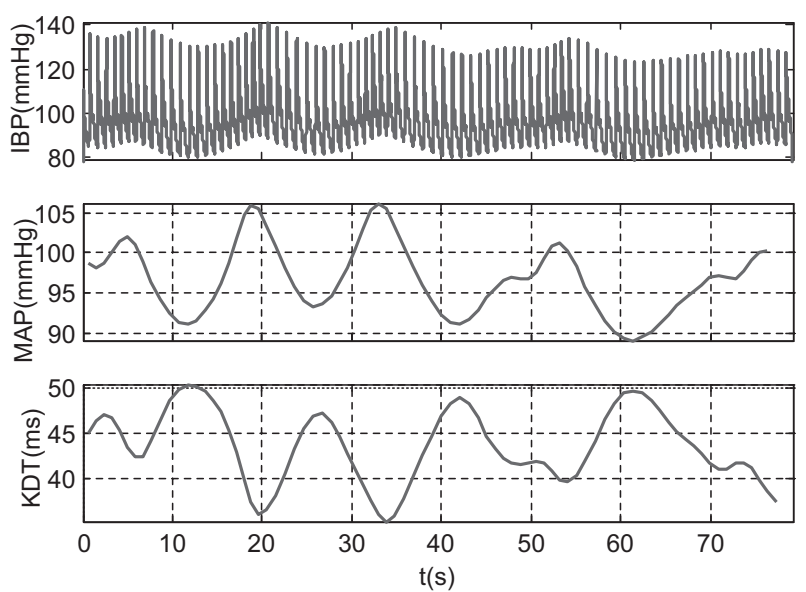

Fig. 4. The invasive blood pressure and KDT.

a certain cuff pressure ( $g_{p=100}$, as shown in Fig. 3 ) is calculated. The six subjects' average of $g_{p=100}$ is $1.22 \mathrm{~ms} / \mathrm{mmHg}$.

The change in Korotkoff sound delay time reflects the change in brachial arterial pressure. Figure 4 shows an example of the KDTs and the IBP recorded simultaneously in about $80 \mathrm{~s}$. The top plot is the invasive blood pressure. The middle plot is the beat-to-beat mean arterial pressure obtained by averaged the IBP in a cardiac cycle. The bottom plot is the beat-to-beat KDT. The fluctuations of the invasive blood pressure and KDT are caused by the paced breath of the subject. The correlation coefficient between the MAP and the KDT is 0.90 .

\section{Discussion}

The Korotkoff sound delay time becomes short as the cuff pressure decreases in the blood pressure measurement process. The approximate coefficients $\left(k_{1}\right.$ and $\left.k_{2}\right)$ are observed for repeated measurements on the same subject, but the coefficients of different subjects are not always the same. In fact, each subject has own coefficients of KDT and cuff pressure, especially for those hypertensive or hypotensive 

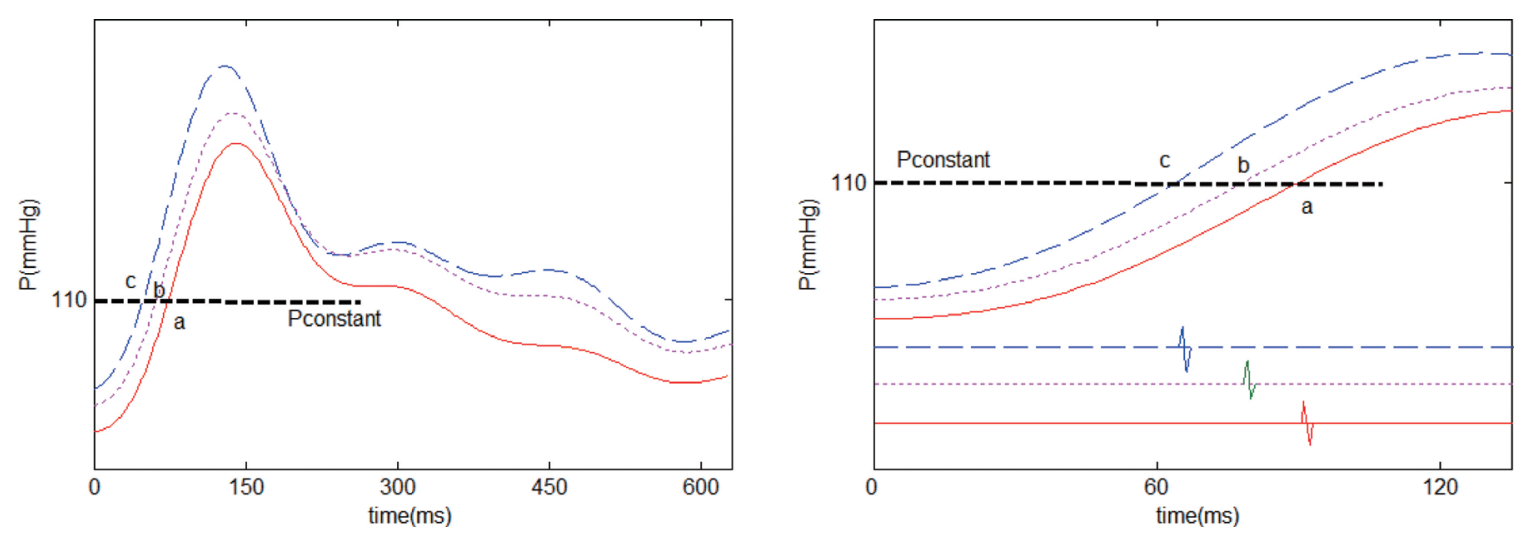

Fig. 5. The fluctuation of KDT induced by the blood pressure fluctuation.

individuals. Furthermore, the curve reflecting the relation between Korotkoff sound delay time and the cuff pressure is similar to the ascend edge of the brachial pressure waveform. These may be a noninvasive means to explore the individuals' cardiovascular status, such as blood pressure, stiffness and peripheral impedance. The advantage of applying Korotkoff sound delay time based on the oscillometric pulse is that there is no need for another reference signal, such as ECG, which hence facilitate the application of the KDT.

The change in KDT associates the change in brachial arterial pressure while the cuff pressure keeps constant approximately (shut off the deflating valves). As shown in Fig. 5. While the cuff pressure and the arterial pressure keeps stable, the Korotkoff sound will occur at the same time on the ascending edge of the arterial pulse for each cardiac cycle and the KDT will not change. In Fig. 5, the cuff pressure keeps at $110 \mathrm{mmHg}$. The cross points of the constant cuff pressure and the three arterial pressure waveform were labeled with $\mathrm{a}, \mathrm{b}$ and $\mathrm{c}$ from the lower pressure to the higher pressure. The cross points locate at the ascending edge. From Fig. 5, we can find that the lower arterial pressure corresponds to a longer Korotkoff sound delay time (cross point a). With the arterial pressure increasing, the cross points appear more and more early in the ascending edge of the arterial pressure waveform. While describing the relationship between KDT and the cuff pressure as shown in Fig. 1, the brachial arterial pressure is assumed stable in the whole measurement process. On the other way, while the pressure in the cuff keeps stable, the change in the brachial arterial pressure will lead to the change in transmural pressure of the arterial wall. Consequently, the KDT will change with the brachial arterial pressure. In fact, the KDT is a function of transmural pressure of the brachial arterial wall. Either the change in the arterial pressure or in the cuff pressure will lead to the change in transmural pressure. Thus, the KDT will change correspondingly. These characteristics may be applied to estimate the compliance of the artery.

The Korotkoff sound delay time utilizing the ECG as the timing reference includes three intervals: the pre-ejection period (PEP), the pulse transit time (PTT) from aortic to brachial and the delay time in the brachial under the cuff [14]. The variation of the Korotkoff sound due to the blood pressure is distorted by the PEP [15]. Hence, utilizing the distorted KDT with ECG $R$ wave or $Q$ wave as reference to measure the blood pressure may reduce its credibility. However, the oscillometric pulse in the cuff is a reasonable reference to determine the KDT, which is easy to eliminate the interference of the PEP.

The KDT can be employed to calibrate the pulse transit time method in blood pressure measurement. The KDT can be used to estimate the blood pressure noninvasively if the cuff pressure is kept between systolic and diastolic pressure. Furthermore, combined with the simultaneous pulse transit time, the 
calibrated procedure with KDT can individualize the measurement and hence enhances the accuracy of blood pressure measurement in PTT method [16].

The other potential applications of the Korotkoff sound delay time in blood pressure measurement include head-up tilt (HUT), cold pressor test (CPT) and cardiopulmonary function test [17/18]. The Korotkoff sound delay time is also applicable to the temporal artery on the head to explore the change in blood pressure of the pilots' cerebral vascular in high $+\mathrm{Gz}$ acceleration maneuvers. A bladder can be applied over the temporal artery on the head to occlude the artery to obtain Korotkoff sounds similar with the traditional Korotkoff method on the brachial artery [19|20]. The Korotkoff sound delay times for each heart beats can be employed to estimate the beat-to-beat blood pressure in the artery on the head during the acceleration training course and evaluate the $+\mathrm{Gz}$ tolerance in aviation medicine.

\section{Conclusion}

The occurrence time of the Korotkoff sound demonstrates characteristic during the cuff deflating process of blood pressure measurement. The Korotkoff sound delay time (KDT) decreases as the cuff pressure deflates. KDT is a function of arterial transmural pressure and its characteristic might be used to measure the mechanical character of brachial artery and the blood pressure noninvasively.

\section{Acknowledgments}

This work is partially supported by National Nature Science Foundation of China Grant 81271737 , 30800238 and National Key Technology R\&D Program Grant 2013BAI03B02, 2013BAI03B02.

\section{References}

[1] Shevchenko YL and Tsitlik JE. 90th Anniversary of the development by Nikolai S. Korotkoff of the auscultatory method of measuring blood pressure. Circulation. 1996; 94(2): 116-118.

[2] Drzewiecki GM, Melbin J, and Noordergraaf A. The Korotkoff sound. Ann Biomed Eng. 1989; 17(4): 325-359.

[3] Mccutcheon EP, Rushmer RF, Jacobson O, and Sandier H. Korotkoff sounds: An experimental critique. Circulation Research. 1967; 20(2): 149-161.

[4] Wang Y, She J, Xiang H, Li Y, Liu J, Li D et al. Improving auscultatory blood pressure measurement with electronic and computer technology: The visual auscultation method. American Journal of Hypertension. 2009; 22(6): 624-629.

[5] Allen J, Gehrke T, O'Sullivan JJ, King ST, and Murray A. Characterization of the Korotkoff sounds using joint time frequency analysis. Physiol Meas. 2004; 25(1): 107.

[6] Gosse P, Cailleau C, Barthelemy JC, Chevalier JM, and Clementy J. Ambulatory measurement of Korotkoff sounds timing (QKD interval) in a normal population. Arch Mal Coeur Vaiss. 1994; 87(8): 1083-1086.

[7] Rodbard D, Fujita T, and Rodbard S. Estimation of thyroid function by timing the arterial sounds. JAMA. 1967; 201(11): 884-887.

[8] Ng KG and Small CF. Survey of automated noninvasive blood pressure monitors. J Clin Eng. 1994; 19(6): 452-475.

[9] Xiang H, Liu Y, Li Y, Qin Y, and Yu M. Noninvasive measurement of beat-to-beat arterial blood pressure by the Korotkoff sound delay time. Blood Pressure Monitoring. 2012; 17(1): 35-41.

[10] Brookman B, Dalton C, and Geddes L. The relationship between vessel-wall elasticity and Korotkoff-sound frequency. Medical and Biological Engineering. 1970; 8(2): 149-158.

[11] Ware R and Anderson W. Spectral analysis of Korotkoff sounds. Biomedical Engineering, IEEE Transactions on. 1966; (4): $170-174$.

[12] Gupta R, Miller JW, Yoganathan AP, Udwadia FE, Corcoran WH, and Kim BM. Spectral analysis of arterial sounds: A noninvasive method of studying arterial disease. Medical and Biological Engineering. 1975; 13(5): 700-705.

[13] Maurer AH and Noordergraaf A. Korotkoff sound filtering for automated three-phase measurement of blood pressure. American Heart Journal. 1976; 91(5): 584-591. 
[14] Arzbaecher RC and Novotney RL. Non-invasive measurement of the arterial pressure contour in man. Bibl Cardiol. 1973; 0(31): 63-69.

[15] Payne RA, Symeonides CN, Webb DJ, and Maxwell SR. Pulse transit time measured from the ECG: An unreliable marker of beat-to-beat blood pressure. J Appl Physiol (1985). 2006; 100(1): 136-141.

[16] Xiang H, Liu Y, Qin Y, Pan W, and Yu M, Calibration of pulse wave transit time method in blood pressure measurement based on the korotkoff sound delay time, World Congress on Medical Physics and Biomedical Engineering May 26-31, 2012, Beijing, China, pp. 426-429.

[17] Forleo C, Guida P, Iacoviello M, Resta M, Monitillo F, Sorrentino S et al. Head-up tilt testing for diagnosing vasovagal syncope: A meta-analysis. Int J Cardiol. 2013; 168(1): 27-35.

[18] Bellinazzi VR, Sposito AC, Schreiber R, Mill JG, Krieger JE, Pereira AC et al. Response to cold pressor test predicts long-term changes in pulse wave velocity in men. Am J Hypertens. 2014; 27(2): 157-161.

[19] LaCourse J, Sivaprasad K, and Rogers D. A system to measure a pilot's temporal pulse pressure during acceleration. Aviation, Space, and Environmental Medicine. 1991; 62(4): 356-362.

[20] Shenoy D, von Maltzahn WW, and Buckey JC. Noninvasive blood pressure measurement on the temporal artery using the auscultatory method. Ann Biomed Eng. 1993; 21(4): 351-360. 